\title{
Bayesian model updating with consideration of modeling error
}

\author{
Erliang Zhang — Pierre Feissel — Jérôme Antoni \\ Laboratoire Roberval de Mécanique, UMR 6253 \\ Université de Technologie de Compiègne, France \\ erliang.zhang, pierre.feissel, jerome.antoni@utc.fr
}

ABSTRACT. On account of measurement and modeling errors, structural identification is better tackled within the statistical framework. In this work, a complete process of Bayesian inference for the characterization of the dynamic behavior of a linear structure is presented in the frequency domain. The polynomial chaos expansion is adopted as a surrogate model to propagate the parameter uncertainty and thus accelerate the evaluation of their posterior probability distribution. Moreover, one hybrid modal model is proposed by introducing some additional variables so as to deal with the modeling errors. Bayesian updating is validated experimentally on a steel square plate and the proposed hybrid modal model is illustrated numerically on a cantilever beam.

RÉSUMÉ. En raison des erreurs de mesure et de modélisation, l'identification des paramètres d'une structure est mieux traitée dans un cadre probabiliste. Dans cet article, un processus complet d'inférence bayésienne pour caractériser le comportement dynamique d'une structure linéaire est présenté dans le domaine fréquentiel. Le chaos polynomial est adopté pour propager l'incertitude des paramètres et ainsi accélérer l'évaluation de la distribution de probabilité a posteriori. De plus, un modèle modal hybride est proposé par l'introduction de variables supplémentaires de sorte à traiter l'erreur de modélisation. Le recalage bayesien est validé expérimentalement sur une plaque carrée en acier et le modèle modal hybride proposé est illustré numériquement sur une poutre encastrée.

KEYWORDS: bayesian inference, modeling errors, polynomial chaos, hybrid modal model.

MOTS-CLÉS : inférence bayésienne, erreur de modélisation, chaos polynomial, modèle modal hybride.

DOI:10.3166/EJCM.19.255-266 (C) 2010 Lavoisier, Paris

EJCM - 19/2010. Giens 2009, pages 255 to 266 


\section{Introduction}

The investigation of structural dynamic behavior is a crucial phase to understand and control structures. On the one hand, the experimental modal analysis (Fu et al., 2001) allows the characterization of structures in a given measured frequency band and at given measurement positions, but it is not originally designed as a predictive approach. On the other hand, the numerical model most widely used, Finite Element (FE) model, can be predictive in a certain frequency band (e.g. low and medium for many types of structures). In this latter case the prior discrepancy between the model predictions and the experimental responses results from the following sources:

1) the model parameter error,

2) the modeling error,

3) the measurement noise and the systematic experimental errors.

The technique of model updating is classically used to decrease the distance between the model and the reality by adjusting the model parameters, which is reviewed in several references (Mottershead et al., 1993), (Friswell et al., 1995) and (Natke, 1998). The error 2) is due to the unavoidable simplification to the real structure during the modeling process ; along with error 3), it makes the tuning of model parameters hard to perform. Besides, the model updating has often to face the issue of non-idenfiability or multi-modality. The problem of non-identifiability can be solved by combining more information, for instance via the Tikhonov regularization (Calvettia et al., 2000); however, it still remains in this kind of approach the difficulty of the choice of the implemented distance as well as the weight between the residual term and the regularization term. To answer these difficulties, the Bayesian method (Tarantola, 2005) offers a framework which makes possible the combination of all the prior information to build the cost function in an optimal way. Moreover, by expressing the information on the variables of interest (model parameters, measurement noise and modeling errors) in terms of probability distributions, the Bayesian approach provides a posterior information much richer than an optimal deterministic solution.

In this work, the FE model updating within the Bayesian framework will be carried out by considering all the three types of errors listed above. Section 2 introduces a Bayesian framework for model updating in the frequency domain where the polynomial chaos expansion is used to represent the stochastic FE model and the modeling error is considered, in a standard way, along with the measurement noise. The consideration of the modeling error is in fact a difficult task, on which depends the effectiveness of the method of model updating and the identification results. Section 3 proposes a hybrid modal model to take into account more accurately the deficiency of the FE model ; the update of model parameters and the quantification of the associated modeling errors are then tackled simultaneously with the Bayesian inference. Section 4 is dedicated to a sampling tool, Monte Carlo Markov Chain (Hastings, 1970), for the exploration of the posterior probability function. Finally, section 5 illustrates the 
proposed methodology on an experimental steel plate under free-free condition and on a numerical cantilever beam.

\section{Model updating within the Bayesian framework}

The FE model updating being seen as an inverse problem, the forward problem and the measurement are firstly presented. Systems with single-input and multiple-output $\left(n_{s}>1\right)$ are considered in this work.

\subsection{Forward problem and measurements}

For a linear structure, its dynamic behavior is governed by,

$$
[M(\boldsymbol{\theta})] \ddot{x}(t)+[C(\boldsymbol{\theta})] \dot{x}(t)+[K(\boldsymbol{\theta})] x(t)=\mathbf{F}(t)
$$

where the mass matrix $[M(\boldsymbol{\theta})]$, the dissipation matrix $[C(\boldsymbol{\theta})]$ and the stiffness matrix $[K(\boldsymbol{\theta})]$ are in $\mathbb{R}^{n \times n}$ with $n$ the number of degrees of freedom of the discretized structure $; \mathbf{F} \in \mathbb{R}^{n \times 1}$ is the vector nodal force with one non-zero element ; $x$ is the vector of nodal displacements, and $\boldsymbol{\theta}$ is the vector of model parameters to be tuned, which usually reflect geometrical, material and boundary condition properties. The damping modeling is a difficult issue in structural dynamics. However, the dissipation matrix of a lightly damped structure can be reasonably assumed to be diagonalized by the eigenvectors of the corresponding undamped system (Gawronski et al., 1997). In the proposed study, the diagonal damping coefficients are identified by experimental modal analysis prior to the Bayesian inference. Nevertheless, the proposed approach taking the modeling error into account, once validated on the current study, might be extended to the treatment of damping that might be very uncertain for a complex mechanical system (Pellissetti et al., 2008).

Let us convert Equation [1] in the frequency domain which, compared to the time domain, offers several advantages: it significantly simplifies the identification task since it naturally restricts the analysis to the frequency band of interest; it endows the measurement noise with a circular complex Gaussian distribution according to the central limit theorem (Rice, 1995) and thus allows the level of the measurement noise to be measured experimentally (Pintelon et al., 2001). In the stationary state, the Fourrer transform of Equation [1] yields $\mathbf{U}(\omega)=\mathbf{H}(\omega) \mathbf{F}(\omega)$ with $\mathbf{U}(\omega)=-\omega^{2} \mathbf{X}(\omega)$. For the $i^{\text {th }}$ element of the vector $\mathbf{H}(\omega)$,

$$
\mathbf{H}_{i}(\omega)=\sum_{r=1}^{n_{m}} \frac{-\omega^{2} \mathcal{A}_{r}^{(i)}}{\omega_{r}^{2}-\omega^{2}+2 j \eta_{r} \omega_{r} \omega}
$$

with $j^{2}=-1, n_{m}$ the number of modes in the frequency band of interest and $\eta_{r}$ the diagonal damping coefficients. The transfer function describes very compactly the linear structural dynamic behavior in the frequency domain: its parameters - natural 
frequencies $\omega_{r}$, and modal residues $\mathcal{A}_{r}$ arranged in a vector $\boldsymbol{\alpha}$ are returned by the FE package given the model parameters $\boldsymbol{\theta} . \boldsymbol{\alpha} \in \mathbb{R}^{n_{\alpha} \times 1}$ with $n_{\alpha}$ the dimension of the vector $\boldsymbol{\alpha}$. As illustrated in Figure 1, the relation between the $i^{\text {th }}$ measured output and the corresponding model prediction based on an additive error model is,

$$
Y_{i}(\omega)=U_{i}(\omega)+N_{i}(\omega), \quad U_{i}(\omega)=H_{i}(\boldsymbol{\alpha}, \omega) F(\omega)
$$

where $F(\omega)$ and $Y_{i}(\omega)$ stand for the input load and the response of the system, respectively; the additive error $N_{i}(\omega)$ normally endows both measurement errors and the lack of model accuracy.

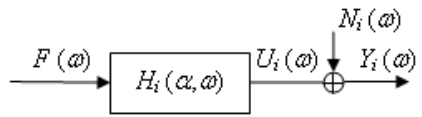

Figure 1. Input-output relation with the additive error model

\subsection{Bayesian formulation for model updating}

In the Bayesian setting, prior and posterior probabilities represent the degree of belief about possible values of model parameters before and after observing the data $[Y]=\left[\mathbf{Y}_{1} ; \cdots ; \mathbf{Y}_{n_{s}}\right]$ with $\mathbf{Y}_{i}$ the vector of structural response at the $i^{\text {th }}$ position over the frequency band of interest. The posterior probability distribution $p\left(\boldsymbol{\theta}, \sigma^{2} \mid \mathcal{D}\right)$ with $\mathcal{D}$ the set of the experimental observations $[Y]$ and the known parameters of the prior probability distribution, is the full solution of the Bayesian approach, which is defined according to Bayes' rule by merging together the available prior information and experimental information from the observed data,

$$
\begin{aligned}
p\left(\boldsymbol{\theta}, \sigma^{2} \mid \mathcal{D}\right) & =p\left([Y] \mid \boldsymbol{\theta}, \sigma^{2}\right) p(\boldsymbol{\theta}) p\left(\sigma^{2}\right) / p(\mathcal{D}) \\
& \propto p(\boldsymbol{\theta}) p\left(\sigma^{2}\right) \prod_{i=1}^{n_{s}} p\left(\mathbf{Y}_{i} \mid \boldsymbol{\theta}, \sigma^{2}\right)
\end{aligned}
$$

where $p(\boldsymbol{\theta})$ is the prior probability distribution of the model parameters and $p\left(\sigma^{2}\right)$ the prior probability distribution of the modeling error. The vector $\boldsymbol{\theta}$ of model parameters is obviously independent of $p(\mathcal{D})$ which can thus be omitted from the formulation. The likelihood function $p\left([Y] \mid \boldsymbol{\theta}, \sigma^{2}\right)$ carries the experimental information and describes the distance $N_{i}(\omega)$ in Figure 1, under the assumption that the modeling error, one part of $N_{i}(\omega)$, is a circular complex Gaussian distribution with variance $\sigma^{2}$. Considering a set of frequency lines $\left\{\omega_{k}\right\}_{k=1}^{n_{\omega}}$, it can be expressed

$$
p\left(\mathbf{Y}_{i} \mid \boldsymbol{\theta}, \sigma^{2}\right)=\frac{1}{\pi^{n_{\omega}} \prod_{k=1}^{n_{\omega}} \sigma_{N_{i}}^{2}\left(\omega_{k}\right)} \exp \left(-\left\|\left(\mathbf{Y}_{i}-\left\lceil H_{i}(\boldsymbol{\alpha}(\boldsymbol{\theta}))\right\rfloor \mathbf{F}\right) / \sigma_{N_{i}}\right\|^{2}\right)
$$


with $\left\lceil H_{i}(\boldsymbol{\alpha}(\boldsymbol{\theta}))\right\rfloor=\operatorname{diag}\left(H_{i}\left(\boldsymbol{\alpha}(\boldsymbol{\theta}), \omega_{1}\right), H_{i}\left(\boldsymbol{\alpha}(\boldsymbol{\theta}), \omega_{2}\right), \cdots, H_{i}\left(\boldsymbol{\alpha}(\boldsymbol{\theta}), \omega_{n_{\omega}}\right)\right)$.

The updated posterior information on $\boldsymbol{\theta}$ and $\sigma^{2}$ can be extracted from the posterior probability distribution $p\left(\boldsymbol{\theta}, \sigma^{2} \mid \mathcal{D}\right)$, which will be stated in more details in Section 4 . However, such operation of extraction is time-consuming, even prohibitive for large scale structures, because it involves the expensive FE calculations to be repeated in the mapping from $\boldsymbol{\theta}$ to $H_{i}(\boldsymbol{\alpha}(\boldsymbol{\theta}))$. In order to keep propagation of uncertainty through the forward model realistic from a computational point of view, a spectral stochastic FE model (Ghanem et al., 1991) is constructed as a surrogate model to replace the calculation of the vector $\boldsymbol{\alpha}$ of modal parameters including natural frequencies and modal residues.

\subsection{Surrogate model}

For any $L_{2}$ random variable, the polynomial chaos (Wiener, 1938), (Ghanem et al., 1991) allows its expansion from a set of independent standardized Gaussian variables $\boldsymbol{\xi}$. The Hermite polynomial chaos expansion then consisting in approximating the modal parameters (forward problem) vector $\alpha$ in the following truncated form,

$$
\boldsymbol{\alpha}(\boldsymbol{\xi}) \approx \sum_{k=1}^{P} \mathbf{a}_{k} \Psi_{k}(\boldsymbol{\xi})
$$

where $\mathbf{a}_{k} \in \mathbb{R}^{n_{\alpha} \times 1}, \Psi_{k}(\boldsymbol{\xi})$ is a Hermite polynomial with $\boldsymbol{\xi}$ the standard normal random vector, and $P$ is the order of the truncated development such that $P=\frac{\left(n_{\xi}+p\right) !}{n_{\xi} ! p !}$ with $n_{\xi}$ the dimension of $\boldsymbol{\xi}$ and $p$ the degree of the Hermite polynomial. The causality between the modal parameters $\boldsymbol{\alpha}$ and the random vectors $\boldsymbol{\xi}$ are created by expressing the model parameters $\boldsymbol{\theta}(\boldsymbol{\xi})$ as a function of $\boldsymbol{\xi}$.

The property of Hermite polynomials to be orthogonal with respect to the standardized Gaussian distribution can be used to determine the coefficient in Equation [6],

$$
\mathbf{a}_{k}=\frac{\left\langle\boldsymbol{\alpha}(\boldsymbol{\xi}) \Psi_{k}(\boldsymbol{\xi})\right\rangle}{\left\langle\Psi_{k}^{2}(\boldsymbol{\xi})\right\rangle}, \text { with }\left\langle\boldsymbol{\alpha}(\boldsymbol{\xi}) \Psi_{k}(\boldsymbol{\xi})\right\rangle=\int \boldsymbol{\alpha}(\boldsymbol{\xi}) \Psi_{k}(\boldsymbol{\xi}) p(\boldsymbol{\xi}) \mathrm{d} \boldsymbol{\xi}
$$

with $p(\boldsymbol{\xi})$ the standardized multivariable Gaussian distribution. In this work, a nonintrusive approach is adopted based on FE package in order to evaluate the inner product $\left\langle\boldsymbol{\alpha}(\boldsymbol{\xi}) \Psi_{k}(\boldsymbol{\xi})\right\rangle$ by regression (Iman, 1999) or Gauss-Hermite quadrature (Jedrzejewski, 2005), thus requiring repeated evaluations of $\boldsymbol{\alpha}$ corresponding to different realizations of $\boldsymbol{\xi}$.

The vector $\boldsymbol{\alpha}$ of unknown modal parameters being parameterized by $\boldsymbol{\xi}$, the polynomial chaos representation works as a surrogate model of the FE model, which allows the propagation of uncertainty from the $\xi$-space to the $\alpha$-space. The function $\theta(\xi)$ 
having been constructed according to the prior distribution of $\boldsymbol{\theta}$, the Bayesian formula of Equation [4] can be converted into the $\boldsymbol{\xi}$-space,

$$
p\left(\boldsymbol{\xi}, \sigma^{2} \mid \mathcal{D}\right) \propto p(\boldsymbol{\xi}) p\left(\sigma^{2}\right) \prod_{i=1}^{n_{s}} p\left(Y_{i}(\omega) \mid \boldsymbol{\xi}, \sigma^{2}\right) .
$$

It is worth pointing out once again that the surrogate model of $\boldsymbol{\alpha}(\boldsymbol{\xi})$ now replaces the FE model in evaluating posterior probability, which makes the sampling particularly inexpensive and therefore drastically accelerate the exploration.

\section{Alternative approach: consideration of the modeling error}

The description of modeling errors is a difficult issue. The standard way in the Bayesian approach is to include it in the additive error $N_{i}(\omega)$ (see Equation [3]), as done in the previous section through a complex circular Gaussian distribution. Nevertheless, such a choice leads to inaccurate results when the modeling error is too large, even after the model has been updated, as it is only partially compensated by the adjustment of the model parameters. Furthermore, the prediction based on the updated transfer function remains in the scope of the FE model, that might be too restrictive. It is therefore of interest to take into account the modeling error in a different way such that the prediction is less restrictive. The reference (Soize, 2005) presents a non-parametric approach that encompasses both the parameter uncertainty and the modeling error by building random mass and stiffness matrices, where the dispersion level of the random matrix are governed by only one parameter. Here, a hybrid formulation of modal parameters is proposed to describe the dynamic behavior based on a stochastic FE model, the idea being to introduce some additional variables corresponding to the modeling error. The objective is not only to update the FE model, but also to quantify the associated posterior modeling error.

\subsection{Additional variables}

Some additional random variables $\epsilon$ are introduced to originally represent the modeling error including the simplification error, the discretization error, the truncation error of polynomial chaos expansion, and so on. The introduced variables $\epsilon$ are separated into $\epsilon_{\mathcal{A}}$ and $\epsilon_{\omega}$ that represent model deficiency on the modal residues and natural frequencies, respectively:

$$
\boldsymbol{\epsilon}=\left[\boldsymbol{\epsilon}_{\omega} ; \boldsymbol{\epsilon}_{\mathcal{A}}\right], \text { with } \boldsymbol{\epsilon}_{\mathcal{A}}=\left[\boldsymbol{\epsilon}_{\mathcal{A}}^{(1)}, \boldsymbol{\epsilon}_{\mathcal{A}}^{(2)}, \cdots, \boldsymbol{\epsilon}_{\mathcal{A}}^{\left(n_{s}\right)}\right]
$$

where $\boldsymbol{\epsilon}_{\omega} \in \mathbb{R}^{n_{\omega}^{d} \times 1}$ and $\boldsymbol{\epsilon}_{\mathcal{A}}^{(i)} \in \mathbb{R}^{n_{\mathcal{A}}^{d} \times 1}$ related to the $i^{\text {th }}$ transfer function. Faced with the difficulty of specifying judiciously its probability distribution, a first straightforward choice is to consider that $\epsilon$ follows a Gaussian distribution,

$$
p(\boldsymbol{\epsilon})=\mathcal{N}\left(0,\left\lceil C_{\epsilon}\right\rfloor\right)
$$


with $\left\lceil C_{\epsilon}\right\rfloor \in \mathbb{R}^{\left(n_{s} n_{\mathcal{A}}^{d}+n_{\omega}^{d}\right) \times\left(n_{s} n_{\mathcal{A}}^{d}+n_{\omega}^{d}\right)}$. The additional variables $\epsilon$ are assumed independent of the model parameters $\boldsymbol{\theta}$ before Bayesian inference. The random variables $\epsilon_{\mathcal{A}}$ assigned to each mode are moreover supposed to have the same variance for all the positions of the measured outputs. The hybrid formulation of modal parameters is then written as follows,

$$
\widetilde{\boldsymbol{\alpha}}(\boldsymbol{\xi}, \boldsymbol{\epsilon})=\boldsymbol{\alpha}(\boldsymbol{\xi})+\boldsymbol{\epsilon} .
$$

\subsection{Bayesian inference in the presence of modeling error}

The prediction of the FE model is linearized with respect to $\epsilon$ using a Taylor expansion at $\boldsymbol{\epsilon}=\mathbf{0}$, for the $i^{\text {th }}$ output,

$$
\mathbf{U}_{i}(\boldsymbol{\alpha}(\boldsymbol{\xi}), \boldsymbol{\epsilon}) \approx \mathbf{U}_{i}(\boldsymbol{\alpha}(\boldsymbol{\xi}))+\mathbf{E}_{i}(\boldsymbol{\xi}, \boldsymbol{\epsilon}),
$$

where $\mathbf{E}_{i}(\boldsymbol{\xi}, \boldsymbol{\epsilon})=\left[S_{i}(\boldsymbol{\xi})\right] \boldsymbol{\epsilon}$ with $\left[S_{i}(\boldsymbol{\xi})\right] \in \mathbb{C}^{n_{\omega} \times\left(n_{\mathcal{A}}^{d}+n_{\omega}^{d}\right)}$ the sensibility matrix of $\boldsymbol{\epsilon}$. The variables $\boldsymbol{\xi}$ and $\boldsymbol{\epsilon}$ being of different physical origins and mutually independent from a prior point of view, the Bayesian joint inference of all the parameters of interest is achieved from,

$$
p\left(\boldsymbol{\xi}, \boldsymbol{\epsilon},\left\lceil C_{\epsilon}\right\rfloor\right) \propto p([Y] \mid \boldsymbol{\xi}, \boldsymbol{\epsilon}) p(\boldsymbol{\xi}) p\left(\boldsymbol{\epsilon} \mid\left\lceil C_{\epsilon}\right\rfloor\right) p\left(\left\lceil C_{\epsilon}\right\rfloor\right) .
$$

Considering the additional variables as the nuisance parameters, the marginal likelihood function of the variables $\boldsymbol{\xi}$ is finally expressed as follows,

$$
\begin{aligned}
& p\left([Y] \mid \boldsymbol{\xi},\left\lceil C_{\epsilon}\right\rfloor\right)=\int p([Y] \mid \boldsymbol{\xi}, \boldsymbol{\epsilon}) p\left(\boldsymbol{\epsilon} \mid\left\lceil C_{\epsilon}\right\rfloor\right) \mathrm{d} \boldsymbol{\epsilon} \\
& =\int \frac{1}{\pi^{n_{\omega} n_{s}} \prod_{i=1}^{n_{s}} \operatorname{det}\left(\left\lceil C_{N_{i}}\right\rfloor\right)} \exp \left(-\sum_{i=1}^{n_{s}}\left(\mathbf{Y}_{i}-\mathbf{U}_{i}\right)^{H}\left\lceil C_{N_{i}}\right\rfloor^{-1}\left(\mathbf{Y}_{i}-\mathbf{U}_{i}\right)\right) p\left(\boldsymbol{\epsilon} \mid\left\lceil C_{\epsilon}\right\rfloor\right) \mathrm{d} \boldsymbol{\epsilon}
\end{aligned}
$$

where the integral can be performed analytically thanks to linearisation of the transfer function in Equation [12]. One can note that the marginal likelihood function of $\boldsymbol{\xi}$ now takes into account the measurement noise and the modeling error. The application of the Bayesian inference on the hybrid modal model yields,

$$
p\left(\boldsymbol{\xi},\left\lceil C_{\epsilon}\right\rfloor \mid \mathcal{D}\right) \propto p\left([Y] \mid \boldsymbol{\xi},\left\lceil C_{\epsilon}\right\rfloor\right) p(\boldsymbol{\xi}) p\left(\left\lceil C_{\epsilon}\right\rfloor\right)
$$

that now has to be explored.

\section{Exploration of posterior probability distribution}

Compared with deterministic estimation methods, the solutions of the Bayesian approach are not only the posterior maximum and the mean values of the parameters (e.g. $\xi, \boldsymbol{\theta}$ and $\boldsymbol{\alpha}$ ), but also their joint/marginal probability distributions. The estimation 
of any of these quantities is not easily achieved directly or analytically since the posterior probability functions are highly nonlinear and implicit function of the parameters, as shown in Equation [8] and [15], an effective sampling scheme should be used. In this paper, the Markov chain Monte Carlo (MCMC) is adopted to directly simulate drawing samples from the posterior probability distribution $p(\boldsymbol{\xi} \mid \mathcal{D})$ or $p\left(\boldsymbol{\xi},\left\lceil C_{\epsilon}\right\rfloor \mid \mathcal{D}\right)$. MCMC eliminates the need to calculate the posterior normalization factor $P(\mathcal{D})$. In the present work, the sampling efficiency is improved by the fact that samples are rendered inexpensive by the polynomial chaos expansion. Based on the samples from the posterior probability distribution,

- the best one with the highest probability can be chosen as the posterior maximum value since the samples drawn by MCMC are situated in the region of high probability ;

- the integral required for the posterior mean values can now be approximated among the samples based on Monte Carlo integral ;

- the marginal probability density of parameters of interest can be estimated with the help of kernel density estimation or by the conditional marginal density estimator (Chen et al., 2000) in the case of high dimension.

Unfortunately, we have found that the sampling technique of MCMC with regular Metropolis-Hastings scheme explores quite slowly the posterior space of variables $\boldsymbol{\xi}$, which results in unreliable or unstable estimators. An effective MCMC algorithm is specially desired for complex Bayesian model. The evolutionary MCMC (Liang et al., 2001) is here adopted, which works by simulating a population of samples in parallel with a different temperature attached to each sample. The population is updated by mutation, crossover and exchange operations. All these genetic operators are modified for their applications on MCMC to satisfy the invariance or reversibility property of the Markov chain transitions. As a result, evolutionary MCMC has the learning ability of the genetic algorithm as well as the fast mixing ability of parallel tempering (simulated tempering).

In this work, the identified results of Bayesian inference from MCMC will be expressed in terms of confidence interval on transfer functions. For a certain frequency $\omega$, a large number of realizations $H(\boldsymbol{\alpha}(\boldsymbol{\xi}), \omega)$ are calculated based on samples of $\boldsymbol{\xi}$; the Bayesian confidence intervals of $90 \%$ are then deduced using the Chen-Shao's algorithm (Chen et al., 2000), which returns the shortest confidence interval.

\section{Applications}

At first, the FE model of a practical square steel plate is updated based on Equation [8] applied to experimental data; then the approach based on Equation [15] with consideration of modeling error variables is applied to a numerical cantilever beam. 


\subsection{Experimental steel plate}

The experimental system with one input and five outputs is represented in Figure 2(a). The sampling frequency is $3200 \mathrm{~Hz}$. A random multi-sine signal is used to excite the plate, which is defined as,

$$
r(t)=\sum_{k=1}^{n_{\omega}} R_{k} \sin \left(\omega_{k} t+\varphi_{k}\right), \omega_{k}=2 \pi k / T
$$

where the variables $\varphi_{k}$ are independently et identically distributed according to an uniform distribution such that $\mathbb{E}\left(e^{j \varphi_{k}}\right)=0$. The use of such a periodical signal allows the estimation of the mean value and the associated standard deviation of the signal using the robust approach (Pintelon et al., 2001). 50 signals of different phase realizations, each with 52 periods, were drawn. The first two periods were neglected due to the transient effect. The measured output $\mathbf{Y}_{1}$ is plotted in Figure 2(b) in terms of the mean value and the standard deviation.

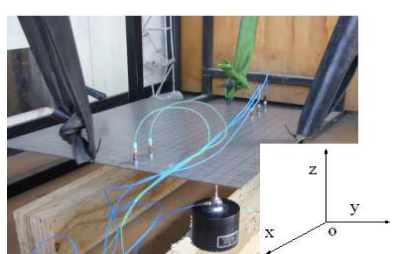

(a) one-input-five-output

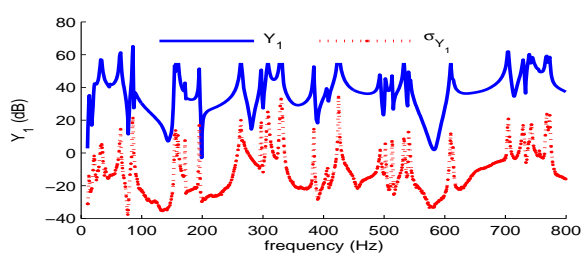

(b) $Y_{1}$ (mean value and standard deviation)

Figure 2. Setup plant and measurement

The plate was discretized into 1600 shell elements using the FE software ANSYS. The thickness $t(\mathrm{~mm})$, the density $\rho\left(\mathrm{kg} / \mathrm{m}^{3}\right)$ and the elastic modulus $E(\mathrm{GPa})$ constitute the vector $\boldsymbol{\theta}$; their prior information was: $E \sim \mathcal{N}(240,30), t \sim \mathcal{N}(2.7,0.1)$, $\rho \sim \mathcal{N}(7500,300) . p\left(\sigma^{2}\right)$ was assumed to be an inverse-Gamma: Inv- $\Gamma(2,5)$. The damping coefficients identified experimentally was on the oder of $10^{-3}$.

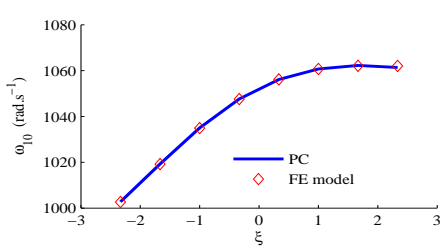

(a) polynomial chaos expansion

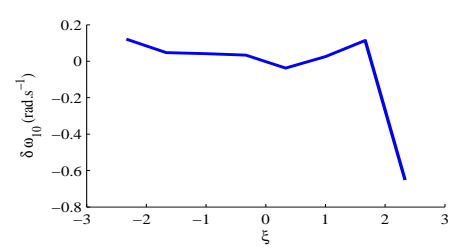

(b) Absolute error

Figure 3. Polynomial chaos expansion of the $10^{\text {th }}$ eigenfrequency $\omega_{10}$ 
The Gausse-Hermite quadrature was used to estimate the coefficients of the polynomial chaos expansion based on the non-intrusive approach, the validity of the polynomial chaos expansion is verified in Figure 3. The uncertainty of prior transfer function stems from the prior uncertainty of model parameters $\boldsymbol{\theta}$ and is illustrated by using the Bayesian confidence interval of $90 \%$ on transfer function $\mathbf{H}_{5}$ in Figure 4(a). The posterior information on transfer function $\mathbf{H}_{5}$ is presented in Figure 4(b) where its dispersion is seen to be greatly reduced. The updated model is validated by transfer function $\mathbf{H}_{3}$ which was not used in the updating process as illustrated in Figure 5.

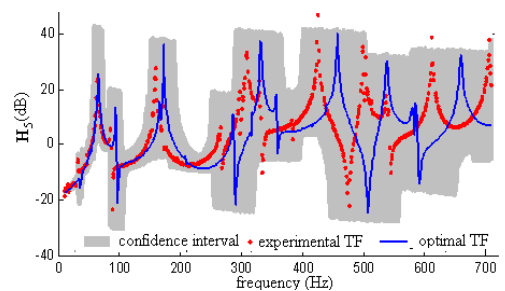

(a) Prior transfer function

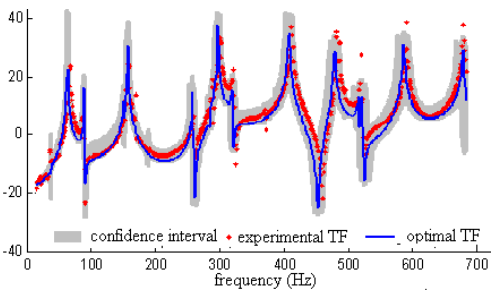

(b) Posterior transfer function

Figure 4. Transfer function $\mathbf{H}_{5}$ (points: experimental TF, line: optimal TF, gray zone: confidence interval)

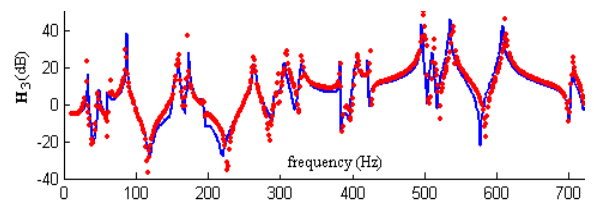

Figure 5. Model validation on $\mathbf{H}_{3}$ (points: experimental TF, line: optimal TF)

\subsection{Numerical cantilever beam}

The proposed hybrid modal model for taking into account the modeling error is applied to a numerical exemple of a cantilever beam, illustrated in Figure 6 . In this example, the modeling errors is caused by discretization errors and artificial errors. The "reality" is simulated by a FE model of 200 beam elements with $E=210.45 \mathrm{GPa}$. Additional artificial errors are added to the modal parameters as follows: $\forall r=1, \cdots, n_{m}, \epsilon_{\omega_{r}}=0.8 \% \omega_{r} x, \quad \epsilon_{\mathcal{A}_{r}}^{(i)}=8 \% \mathcal{A}_{r}^{(i)} x$ where the variable $x \sim \mathcal{N}(0,1), n_{m}=5$. With a sampling frequency of $5000 \mathrm{~Hz}$, the "experimental" response is formed by the "real" system response perturbed by a white Gaussian noise with signal to noise ratio of $38 \mathrm{~dB}$. Two responses $\mathbf{Y}_{1}$ and $\mathbf{Y}_{2}$ illustrated in Figure 6 are used to update our knowledge about $\boldsymbol{\xi}$ and $\left\lceil C_{\epsilon}\right\rfloor$. Response $\mathbf{Y}_{\mathbf{3}}$ is used to validate the updated model. A FE model of 20 elements is used to characterize its dynamic 
behavior, the elastic modulus $E(\mathrm{GPa})$ is introduced as a random variable following the Gaussian law $\mathcal{N}(200,30)$. The inverse Gamma distribution is chosen as the prior probability distribution for $\left\lceil C_{\epsilon}\right\rfloor$, e.g. $\sigma_{\epsilon_{3}}^{2} \sim \operatorname{Inv}-\Gamma(2,5)$. Bayesian confidence interval of $90 \%$ is constructed based on the mean values of $E$ and of $\left[C_{\epsilon}\right]$ for transfer functions $\mathbf{H}_{2}$ as illustrated in Figure 7. The validation of the updated model is shown through transfer function $\mathbf{H}_{3}$ in Figure 8. The prior transfer function is calculated using the prior mean value of $E$.

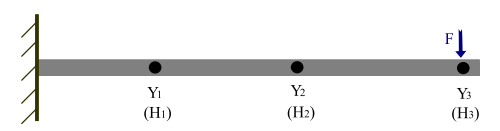

Figure 6. Numerical cantilever beam (measurement plant: one input and three outputs)

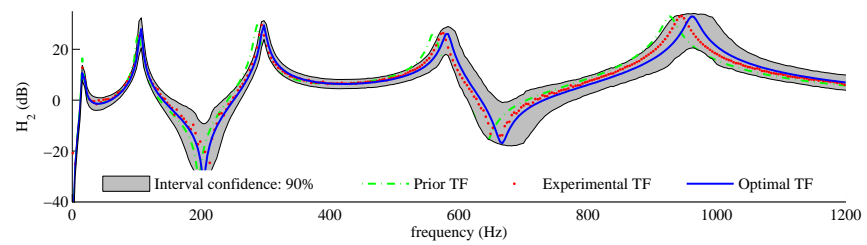

Figure 7. Transfer function $\mathbf{H}_{2}$ (points: experimental TF, dotted line: prior TF, line: optimal TF, gray zone: confidence interval)

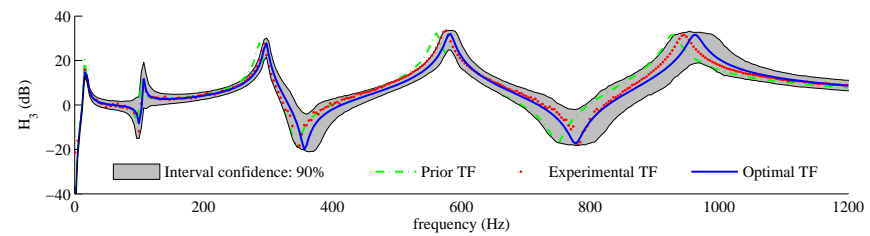

Figure 8. Transfer function $\mathbf{H}_{3}$ (points: experimental TF, dotted line: prior TF, line: optimal TF, gray zone: confidence interval)

\section{Conclusion}

A Bayesian framework for the updating of numerical models of structures from the dynamic responses has been presented in the frequency domain. The Bayesian updating of FE model was carried out based on a surrogate model thanks to the introduction of the polynomial chaos. The consideration of the modeling error being a very difficult problem, it was addressed by introducing additional random variables where the idea 
consisted in better reformulating the distance between the FE model and the reality. Under the simple hypothesis on the introduced variables, the proposed approach was shown promising through a simple numerical example. The more appropriate prior information on the additional random variables is to be further developed.

\section{References}

Calvettia D., Morigib S., Reichelc L., Sgallarid F., « Tikhonov Regularization and the L-curve for Large Discrete Ill-posed Problems », Journal of Computational and Applied Mathematics, vol. 123, p. 423-446, 2000.

Chen M., Q.M.Shao, lbrahim J., Monte Carlo Methods in Bayesian Computation, Springer, 2000.

Friswell M., Mottershead J., Finite Element Model Updating in Structural Dynamics, Springer, 1995.

Fu Z., He J., Modal Analysis, Butterworth-Heinemann Ltd, 2001.

Gawronski W., Sawicki J. T., « Response Errors of Non-Proportionally Lightly Damped Structures », Journal of Sound and Vibration, vol. 200, n 4, p. 543 - 550, 1997.

Ghanem R., Spanos P., Stochastic Finite Elements : A Spectral Approach, Springer, 1991.

Hastings W., « Monte Carlo Sampling Methods Using Markov Chains and Their Applications », Biometrika, vol. 57, p. 97 - 109, 1970.

Iman R., Latin Hypercube Sampling, Wiley-Encyclopedia of Statistical Sciences, 1999.

Jedrzejewski F., Introduction aux Méthodes Numériques, Springer, 2005.

Liang F., Wong W., « Real-Parameter Evolutionary Monte Carlo With Applications to Bayesian Mixture Models », Journal of the American Statistical Association, vol. 96, n 454, p. 653 666, 2001.

Mottershead J., Friswell M., « Model Updating in Structural Dynamics : A Survey », Journal of Sound and Vibration, vol. 167, n 2, p. 347 - 375, 1993.

Natke H. G., « Problems of Model Updating Procedures : a Perspective Resumption », Mechanical Systems and Signal Processing, vol. 12, n ${ }^{\circ}$ 1, p. 65-74, 1998.

Pellissetti M., Capiez-Lernout E., Pradlwarter H., Soize C., Schuëller G., « Reliability analysis of a satellite structure with a parametric and a non-parametric probabilistic model», Computer Methods in Applied Mechanics and Engineering, vol. 198, n 2, p. 344 - 357, 2008

Pintelon R., Schoukens J., System Identification : A Frequency Domain Approach, Wiley-IEEE Press, 2001

Rice J., Mathematical Statistics and Data Analysis, Duxbury Press, 1995.

Soize C., « A Comprehensive Overview of a Non-parametric Probabilistic Approach of Model Uncertainties for Predictive Models in Structural Dynamics », Journal of Sound and Vibration, vol. 288, n $\mathrm{n}^{\circ}$ 3, p. 623-652, 2005.

Tarantola A., Inverse Problem Theory and Methods for Model Parameter Etimation, SIAM (Society of Industrial and Applied Mathematics), 2005.

Wiener N., «The Homogeneous Chaos », American Journal of Mathematics, vol. 60, $\mathrm{n}^{\circ} 4$, p. 897 - 936, 1938. 